Physics

Physics Research Publications

Purdue University

Year 2010

\title{
Dynamical transition in proteins and non-Gaussian behavior of low-frequency modes in self-consistent normal mode analysis
}
J. G. Guo
T. Budarz
J. M. Ward
E. W. Prohofsky

This paper is posted at Purdue e-Pubs.

http://docs.lib.purdue.edu/physics_articles/1238 


\title{
Dynamical transition in proteins and non-Gaussian behavior of low-frequency modes in self-consistent normal mode analysis
}

\author{
Jianguang Guo, ${ }^{1}$ Timo Budarz, ${ }^{2}$ Joshua M. Ward, ${ }^{3}$ and Earl W. Prohofsky ${ }^{1, *}$ \\ ${ }^{1}$ Department of Physics, Purdue University, West Lafayette, Indiana 47907, USA \\ ${ }^{2}$ Department of Physical Science, Santa Ana College, Santa Ana, California 92706, USA \\ ${ }^{3}$ Department of Medicinal Chemistry and Molecular Pharmacology, Purdue University, West Lafayette, Indiana 47907, USA
}

(Received 26 May 2010; revised manuscript received 22 July 2010; published 21 October 2010)

\begin{abstract}
Self-consistent normal mode analysis (SCNMA) is applied to heme c type cytochrome $\mathrm{f}$ to study temperature-dependent protein motion. Classical normal mode analysis assumes harmonic behavior and the protein mean-square displacement has a linear dependence on temperature. This is only consistent with lowtemperature experimental results. To connect the protein vibrational motions between low and physiological temperatures, we have incorporated a fitted set of anharmonic potentials into SCNMA. In addition, quantum harmonic-oscillator theory has been used to calculate the displacement distribution for individual vibrational modes. We find that the modes involving soft bonds exhibit significant non-Gaussian dynamics at physiological temperature, which suggests that it may be the cause of the non-Gaussian behavior of the protein motions probed by elastic incoherent neutron scattering. The combined theory displays a dynamical transition caused by the softening of few "torsional" modes in the low-frequency regime $\left(<50 \mathrm{~cm}^{-1}\right.$ or $<6 \mathrm{meV}$ or $\left.>0.6 \mathrm{ps}\right)$. These modes change from Gaussian to a classical distribution upon heating. Our theory provides an alternative way to understand the microscopic origin of the protein dynamical transition.
\end{abstract}

DOI: 10.1103/PhysRevE.82.041917

PACS number(s): 87.15.ad, 87.15.H-, 87.15.Zg

\section{INTRODUCTION}

Protein function is determined by both structural stability and flexibility. The stability is needed to ensure appropriate geometry of the protein, while the flexibility allows function to proceed at an appropriate rate. Quantitative measurements of the temperature-dependent atomic mean-square displacements (MSDs) are possible by neutron scattering [1-3] and Mössbauer absorption [4-6]. All of these experiments show a "dynamical transition" in hydrated proteins, which is marked by an abrupt MSD increase in the temperature range $160-240 \mathrm{~K}$. It is believed that this dynamical transition is correlated with protein function. Three prominent examples are the myoglobin- $\mathrm{CO}$ binding kinetics [7], electrostatic relaxation in green fluorescent protein [8], and the Arrhenius behavior of the electron transfer rate above the dynamical transition temperature. However, the time scale and the forms of the functionally important atomic modes remain a subject of active discussion [9-11].

Numerous theoretical studies of protein dynamics have been carried out by molecular-dynamics simulations [12-15] and normal mode analysis (NMA) [14,16-20]. NMA requires the use of Maxwell-Boltzmann or Gaussian distributions to describe the probability distributions of individual atoms or chemical bonds. Recently, several authors focused on the study of the non-Gaussian behavior of the total elastic incoherent neutron-scattering (EINS) profile from a protein above dynamical transition temperature [21-25]. It should be noted that the distribution of all-atom MSDs from an EINS profile can still be non-Gaussian even if all atoms individually exhibit Gaussian dynamics. The Gaussian distribution, which is the ground-state probability distribution for the

\footnotetext{
*ewp@purdue.edu
}

quantum harmonic oscillator $(\mathrm{QHO})$, is an appropriate approximation when $\hbar \omega>k T\left(\omega>200 \mathrm{~cm}^{-1}\right)$. In the Gaussian distribution, the atom has maximum probability in the equilibrium position. We find that in all self-consistent theories, the use of a Gaussian distribution results in a molecular structure that will tend to be more rigid than what would be found by a more exact quantum approach. From Newton's second law, the classical harmonic oscillator (low frequency) has highest probability at the edges of the well because the atom moves most slowly near the classical turning points, which is contrary to the Gaussian or ground-state probability distribution. The exact quantum behavior of low-frequency modes would approach the classical displacement. In this paper we explore the role of incorporating the higher quantum vibrational states. This shows a softening of the structure in the correct temperature range.

The material studied by SCNMA is six-coordinate heme c type cytochrome f (cyt f) [26]. The iron normal modes are compared with the nuclear vibrational resonance spectroscopy (NRVS) spectrum [27]. NRVS is uniquely capable of displaying the low-frequency vibrational displacement spectrum of the $\mathrm{Fe}$ atom at the center of the heme as it sees all modes and can give quantitative values for displacements. It is then possible to define low-frequency heme modes that are in agreement with observation with greater accuracy. This is a much more stringent test than most Raman comparisons as Raman displacements cannot be calculated with any accuracy.

SCNMA incorporates nonlinearity into harmonic calculation by thermal-statistically averaging the curvature of the bond potential energies. Because vibrational modes that are not overdamped are detected by Raman and IR, one expects the effective Hamiltonian to be approximately harmonic. SCNMA should therefore be a valid approach. The SCNMA formulation arises from a variational procedure that finds the 
best effective harmonic Hamiltonian by minimizing the free energy. This method is described in detail elsewhere [28]. It has been successfully employed on models with multiple hydrogen stretching bonds such as the helix melting, conformational change in DNA, drug-helix stability, etc. [29-32]. In those papers a Gaussian distribution was used to describe the displacement distribution. In this paper, we will further develop this method to incorporate non-Gaussian distributions into our calculation.

\section{QHO THEORY APPLIED TO INTERNAL ATOMIC BONDS}

\section{A. Displacement distribution of the internal atomic bonds}

SCNMA can incorporate anharmonic effects which alter vibrational frequencies as temperature changes. To do this it must incorporate a model of the anharmonicities in bonds. One assumes potential models for each internal degree of freedom of a bond. For example, stretch degrees are assumed to be represented by a Morse potential. These potential parameters are determined by fitting to refined data of effective force constants and bond length at a given temperature from experimental data. The formulas used to calculate temperature-dependent effective force constants will give a good fit based on minimization of the free energy of the system.

For a biomolecule with $N$ atoms and $M$ internal atomic bonds $M$ is much larger than $N$. Standard NMA will give us $3 N-6$ nonzero normal modes. Their frequencies can be written as $\omega=\left[\omega_{1}, \omega_{2}, \ldots, \omega_{3 N-6}\right]$. The total MSD for frequency $\omega$ can be written as

$$
\left\langle\sum_{i=1}^{n} m_{i} r_{i}^{2}\right\rangle=\frac{\hbar}{2 \omega} \operatorname{coth}\left(\frac{\hbar \omega}{2 k_{B} T}\right) .
$$

Subsequently, the temperature-dependent total mean-square amplitude for the one single internal bond is the sum of all normal mode amplitudes, which can be written as

$$
D^{2}=\sum_{\omega} D_{\omega}^{2}=\sum_{\omega} d_{\omega}^{2} \operatorname{coth}\left(\frac{\hbar \omega}{2 k_{B} T}\right)=\sum_{\omega} \frac{\hbar}{2 \omega} \operatorname{coth}\left(\frac{\hbar \omega}{2 k_{B} T}\right)\left|s_{\omega}\right|^{2},
$$

where $D^{2}$ is the total mean-square amplitude over all frequency modes, $D_{\omega}^{2}$ is the mean-square amplitude contribution, $d_{\omega}^{2}$ is the zero-point mean-square amplitude for frequency $\omega$, and $\left|s_{\omega}\right|^{2}$ is the projection of the normalized eigenvectors at eigenvalues (frequency) $\omega$ onto the massweighted internal coordinates. These amplitudes can repre- sent a linear distance (for stretching bond) or an angular twisting (for angle bend and dihedral bond).

The Hamiltonian for one single internal bond can be written as

$$
\hat{H}=-\frac{\hbar^{2}}{2} \nabla_{q_{\omega}}^{2}+\frac{1}{2} \omega^{2} q_{\omega}^{2}=-\frac{\hbar^{2}\left|s_{\omega}\right|^{2}}{2} \frac{d^{2}}{d u_{\omega}^{2}}+\frac{1}{2\left|s_{\omega}\right|^{2}} \omega^{2} u_{\omega}^{2},
$$

where $q_{\omega}$ is the mass-weighted Cartesian coordinate displacement variables and $u_{\omega}$ is the internal coordinate displacement variable for mode $\omega$. Similar as the one-dimension $\mathrm{QHO}$ problem, the wave function for one particular internal bond at frequency $\omega$ can be written as

$$
\Psi_{n}\left(u_{\omega}\right)=\sqrt{\frac{1}{2^{n} n !}}\left(\frac{1}{2 \pi d_{\omega}^{2}}\right)^{1 / 4} e^{-u_{\omega}^{2} / 4 d_{\omega}^{2}} H_{n}\left(\sqrt{\frac{1}{2 d_{\omega}^{2}}} u_{\omega}\right),
$$

where $H_{n}$ is the Hermite polynomial and $\Psi_{n}$ is the wave function for the $n$th excitation state. Here, we note that the ground-state wave function $\Psi_{0}$ is in fact a Gaussian. The corresponding quantized energy levels are

$$
E_{n}=\hbar \omega\left(n+\frac{1}{2}\right) \text {. }
$$

From the Boltzmann distribution, the displacement distribution of this internal coordinate for mode $\omega$ can be written as

$$
f_{\omega}\left(u_{\omega}\right)=\operatorname{Tr}\left(e^{-H / k_{B} T}\right)=\frac{\sum_{n=0}^{\infty} \exp \left[\frac{-\hbar \omega\left(n+\frac{1}{2}\right)}{k_{B} T} \Psi_{n}^{2}\left(u_{\omega}\right)\right]}{\sum_{n=0}^{\infty} \exp \left[\frac{-\hbar \omega\left(n+\frac{1}{2}\right)}{k_{B} T}\right]},
$$

where $H$ is the Hamiltonian. The joint probability density function for $\omega=\left[\omega_{1}, \omega_{2}, \ldots, \omega_{3 N-6}\right]$ can be subsequently written as

$$
g\left(u_{\omega_{1}}, u_{\omega_{2}}, \ldots\right)=\prod_{\omega} f_{\omega}\left(u_{\omega}\right)
$$

The total displacement is $u=\Sigma_{\omega} u_{\omega}$. Using a transformation of variables,

$$
\left[u=\sum_{\omega} u_{\omega}, u_{\omega_{2}}=u_{\omega_{2}}, u_{\omega_{3}}=u_{\omega_{3}}, \ldots\right],
$$

the total displacement distribution can be obtained as

$$
f(u)=\int_{-\infty}^{\infty} \cdots \int_{-\infty}^{\infty} g\left(u-\sum_{j=2}^{3 N-6} u_{\omega_{j}}, u_{\omega_{2}}, u_{\omega_{3}}, \ldots\right) d u_{\omega_{2}} d u_{\omega_{3}} \cdots u_{\omega_{3 N-6}} .
$$



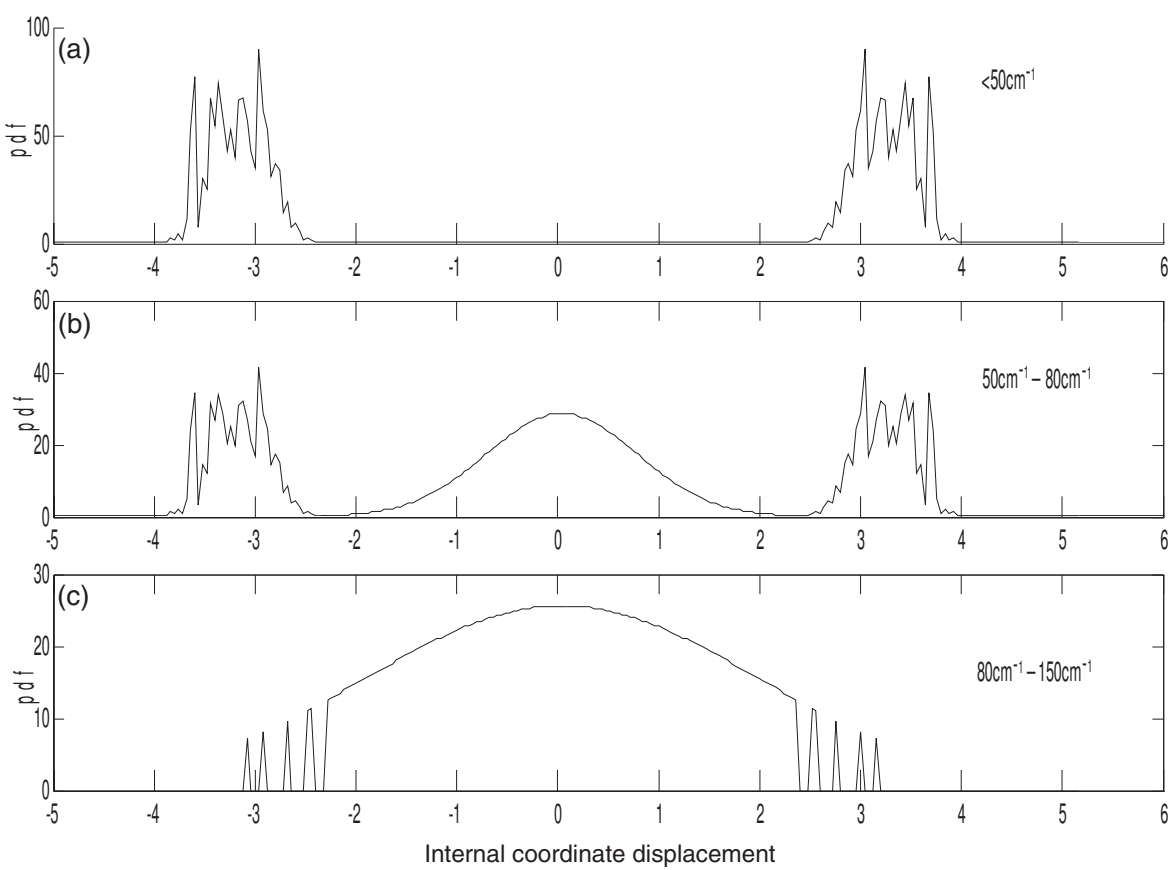

FIG. 1. Characterization of the single frequency displacement distribution at $300 \mathrm{~K}$ in (a) the displacement is similar to the classical distribution, (c) the displacement distribution is similar to a Gaussian, and (b) is a cross between the two. The horizontal axis is displacement variable $u$.
To reduce unsystematic errors, $u_{\omega_{1}}$ is chosen to have the largest amplitude of the $3 N-6$ modes. Equation (9) requires $3 N-7$ integrals of a $3 N-6$ multivariable function to calculate the actual displacement distribution of one single internal bond. For one standard NMA calculation, $(3 N-7) \times M$ integrals are solved. To reduce the required calculation time, approximation methods are employed, as introduced in the next section.

\section{B. Displacement distribution for single frequency harmonic motion and an approximation method}

To understand the approximate temperature and frequency behavior of non-Gaussian distributions, that of a single frequency normal mode displacement is shown in Fig. 1. It shows the temperature-dependent single frequency displacement distribution at $300 \mathrm{~K}$ for (a) $\omega<50 \mathrm{~cm}^{-1}$ (>0.67 ps), (b) $50<\omega<80 \mathrm{~cm}^{-1}(0.42-0.67 \mathrm{ps})$, and (c) $\omega>80 \mathrm{~cm}^{-1}$.

Figure 1 shows the displacement probability for a single frequency, but the displacement for a single bond is a superposition of many such frequency contributions with different amplitudes. The spread in amplitudes comes from the projection factors $\left(\left|s_{\omega}\right|^{2}\right)$ from Eqs. (2) and (3) which come from the eigenvectors of the various modes. Even for low frequencies, any bond amplitude would be the sum of many distributions like those in Fig. 1, all at different amplitudes from the origin. The central limit theorem (CLT) supposes that a large sum of this kind will add up to a Gaussian distribution. This assumption has been central to all previous calculations using SCNMA. The situation could be quite different, however, if only a few low-frequency modes dominate in the displacement of particular bonds. In such a case, for some range of temperatures, the displacement probability could resemble the plot in Fig. 1(a). We emphasize that the hydrogen bond stretching modes are typically above $100 \mathrm{~cm}^{-1}$ and fall into the Gaussian distribution regime. The bond modes that are softer than the hydrogen stretching bonds, i.e., the torsional motions, may exhibit non-Gaussian behavior at physiological temperature. All proteins have torsional modes, and this effect may be manifested in many proteins.

From Eq. (6), the displacement distribution of the single frequency mode is approximately Gaussian when

$$
\omega(T)>0.27 T \mathrm{~cm}^{-1} \quad(T \text { in kelvin }),
$$

and more classical when

$$
\omega(T)<0.17 T \mathrm{~cm}^{-1} \quad(T \text { in kelvin }) .
$$

From Eqs. (10) and (11), the single frequency mode in the frequency regime $<50 \mathrm{~cm}^{-1}$ (or $<6 \mathrm{meV}$ or $>0.7 \mathrm{ps}$ ) will transition from a Gaussian to a more classical distribution upon heating from low temperature to room temperature. It should be noted that the prominent "boson peak" (1-3.5 meV or $10-30 \mathrm{~cm}^{-1}$ ) from neutron scattering $[3,16,33]$ or the "doming mode" from NRVS [27] and IR [34] experiments lie in this frequency regime.

To simplify the calculation, we use the assumption that the sum of the independent Gaussian variables is still a Gaussian, and we treat all the normal modes above $80 \mathrm{~cm}^{-1}$ as one Gaussian distribution. Based on CLT, we can further simply the low-frequency displacement distribution calculation. If the displacement $u$ for one internal bond is comprised of many low-frequency modes, we can treat it as a Gaussian. To test how many significant low-frequency $\left(<80 \mathrm{~cm}^{-1}\right)$ modes are needed to be able to use the Gaussian approximation without loss of accuracy, several NMAs and subsequent displacement distribution calculations were run on the heme core. We found less than 5\% deviation from Gaussian in the distribution of $u$ [Eq. (9)] when $u$ has more than five lowfrequency modes, each accounting for more than $10 \%$ of the total potential energy. Implementing these two approximations reduces our calculation time by a factor of more than 100 . 


\section{METHODS}

An initial classical NMA calculation was performed on the six-coordinate heme $\mathrm{c}$ type cytochrome $\mathrm{f}$ using the CHARMM force field [35,36]. An all-atom model [36] was constructed from the x-ray coordinates [Protein Data Bank (PDB) identifier 1EWH [26]]. The model was subjected to force field minimization until the root-mean-square gradient of the potential energy was less than 0.0001 prior to performing a standard normal mode calculation with the VIBRAN facility in CHARMM [35]. The six lowest normal mode frequencies are less than $10^{-4} \mathrm{~cm}^{-1}$ and the seventh lowest frequency is larger than $1 \mathrm{~cm}^{-1}$, which indicates a good convergence of the minimization procedure. The iron displacements were extracted from the normal modes, and we calculated the iron vibrational density of states (VDOS) $[37,38]$.

The bond stretch, angle bend, and dihedral bond force constants from CHARMM were adopted into our NMA and SCNMA programs. The heme force constants and the hemeprotein backbone interaction force constants were refined by comparison with the NRVS spectrum [27]. The method of force field refinement process was described elsewhere [39-41]. The usual procedure is to make a small modification of the force constants and compare the new output to the measured NRVS.

The anharmonic functional forms were chosen from Ref. [42], in which Morse function, harmonic cosine function, and dihedral cosine function were used to describe bond stretch interactions, angle bend interactions, and torsional bond interactions. The three parameters of the Morse potential and dihedral bond potential and the two parameters of angle bend potential can be fitted by the resulting lowtemperature force constants along with data on atom distances and bond strength. The low-temperature force constants are the second derivatives of the anharmonic potentials at the potential-well minimums. The parameters for the stretching bond strength, which is directly related to the dissociation energy, and that of dihedral bond strength (multiplicity) were obtained from the CHARMM program.

SCNMA was employed to allow exploration of temperature-dependent changes in force constant and thermal expansion effects [28]. This method has been described in detail elsewhere [28-32], where the Gaussian approximation was used for the displacement variable $u$. The only difference here from the previous SCNMA is the explicit inclusion of non-Gaussian distributions for low-frequency modes. Here, we give a brief description of the computation:

(i) Input the effective force constants (the first iteration uses the force constants refined to experimental data) into the NMA and find the initial normal mode eigenvalues and eigenvectors.

(ii) Calculate each internal coordinate's total mean-square amplitude $D^{2}$ and each normal mode contribution $D_{\omega}^{2}$.

(iii) Calculate each internal coordinate displacement distribution $f(u)$.

(iv) Calculate a new set of the effective force constants.

(v) Iterate to self-consistency.

The calculation converged within 20 iterations.

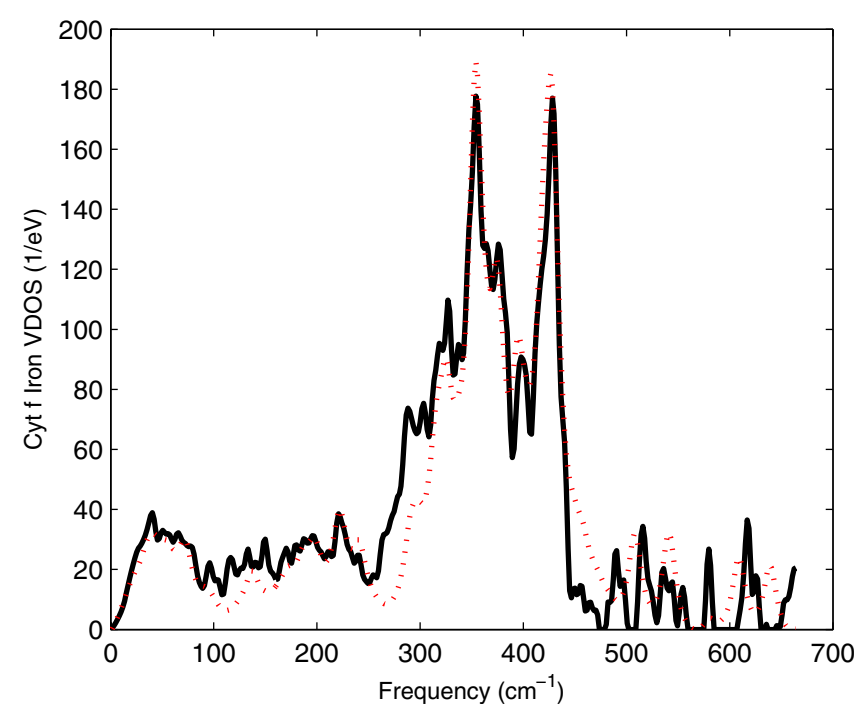

FIG. 2. (Color online) Comparison of the experimental (from Ref. [43]) and theoretical (from classical NMA) cytochrome f iron VDOSs. Solid line: experiment; dotted line: theory.

\section{RESULTS AND DISCUSSION}

Figure 2 shows the comparison between the iron VDOSs obtained from classical NMA and the NRVS experimental results. Good agreement is achieved over a wide range of frequencies, which indicates a useful choice for the lowtemperature limit force field. Here, we give a summary of the general results: (1) below $80 \mathrm{~cm}^{-1}$ are mostly iron out-ofplane motions, (2) the $80-300 \mathrm{~cm}^{-1}$ region has both iron in-plane and out-of-plane features, and (3) $>300 \mathrm{~cm}^{-1}$ are mainly iron in-plane motions. If the calculations did not include anharmonic effects, the total displacements would be linear in temperature. Classical NMA results show that at low temperature $(<150 \mathrm{~K})$, the iron out-of-plane MSD is about three times the iron in-plane MSD despite the fact that the iron in-plane motion has two degrees of freedom versus the single degree of out-of-plane motion.

Figures 3 and 4 show the cyt $\mathrm{f}$ iron total MSD from SCNMA. These results are in general agreement with the Mössbauer absorption experimental results conducted on other heme proteins. The high-frequency $\left(>200 \mathrm{~cm}^{-1}\right)$ normal modes are softened on an average of $1-2 \%$. This is because the high-frequency normal modes are dominated by covalent stretching bonds which have relatively larger strength and deeper potential wells. Moreover, these high-frequency atomic motions follow a strict narrow Gaussian distribution. Figure 4 shows that the iron dynamical transition is caused by iron low-frequency out-of-plane motions. At lower frequency, the large iron out-of-plane motion becomes possible because of the small energy involved in changing the torsion angles. As temperature increases, more and more displacement will spread out from the Gaussian centroid. This lowfrequency classical behavior of the atomic displacement distributions coincides with the fact that the curvature of the potential function decreases over the distance from the centroid, which results in the abrupt MSD increase seen in our SCNMA model as compared with calculations implementing only Gaussian distributions (Fig. 4). 


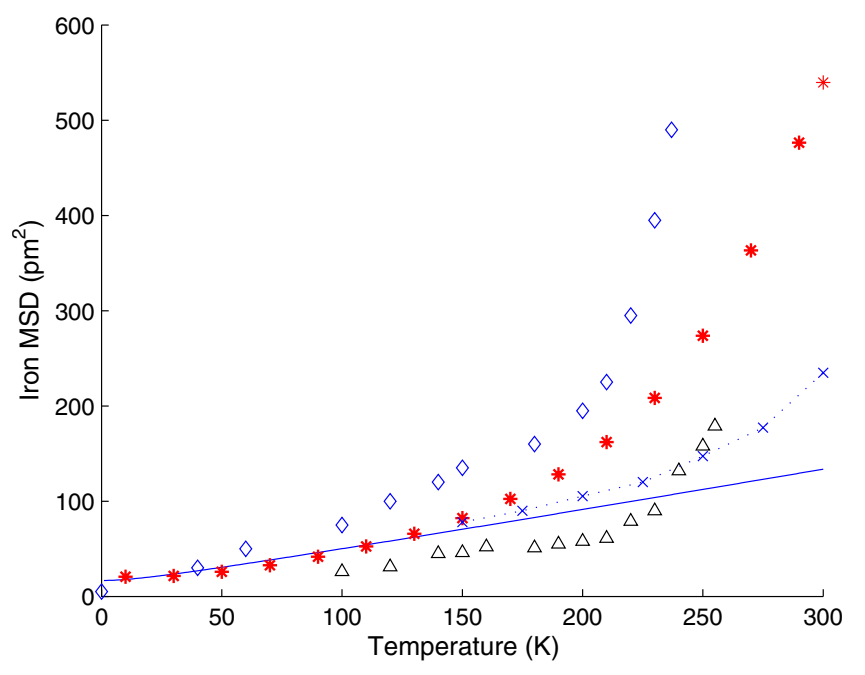

FIG. 3. (Color online) The iron MSD vs temperature plot for various heme proteins. Line: iron MSD of cyt $f$ by classical NMA. Cross and dashed line: iron MSD of cyt $f$ from SCNMA with Gaussian distribution approximation. Star: iron MSD of cyt $f$ from our SCNMA by implementing non-Gaussian displacement distribution. Diamond: iron MSD of myoglobin by Mössbauer absorption measurement from Ref. [4]. Upper triangle: iron MSD of cyt c by Mössbauer absorption measurement from Ref. [5].

To analyze the protein flexibility, the force strength defined by Zaccai [44] is generally used by other authors [45],

$$
k_{0}=\frac{k_{B}}{\frac{d\left\langle r^{2}\right\rangle}{d T}} .
$$

From this definition, the iron force strength decreases by a factor of $\sim 5-7$. From NMA, the force constant is $k_{0}=m \omega^{2}$, and we extract $\omega$ to find $r^{2}$. We found that the dihedral

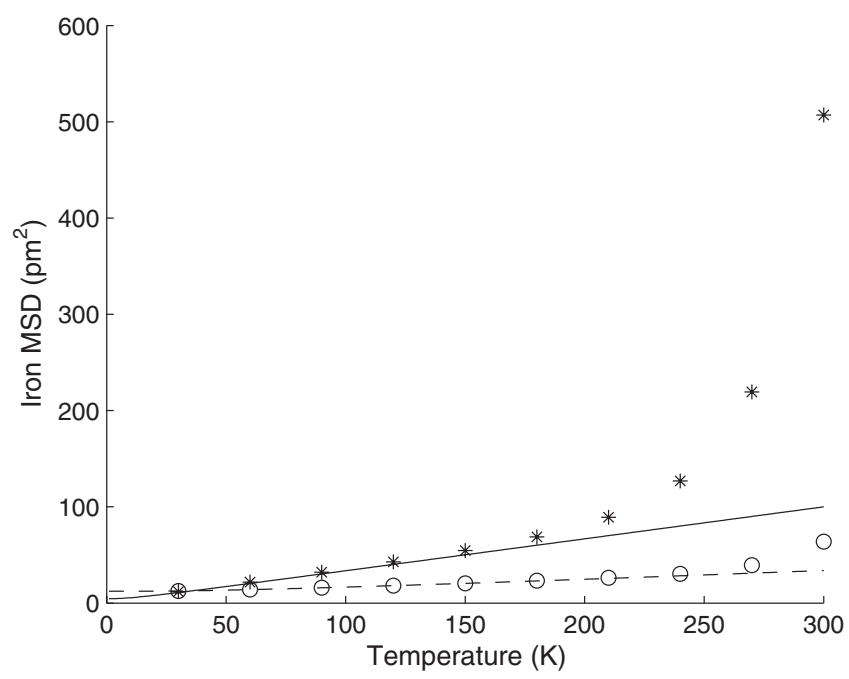

FIG. 4. The cyt $\mathrm{f}$ iron in-plane and out-of-plane MSDs from SCNMA. Dashed line: iron in-plane MSD from classical NMA; circle: iron in-plane motion from SCNMA; solid line: iron out-ofplane motion from classical NMA; star: iron out-of-plane motion from SCNMA.
TABLE I. The softening of the iron dihedral force constant from self-consistent normal mode analysis (SCNMA).

\begin{tabular}{lccc}
\hline \hline Bond type & $\begin{array}{c}<150 \mathrm{~K} \\
\left(\text { mdyn } \AA^{2} / \mathrm{rad}\right)\end{array}$ & $\begin{array}{c}300 \mathrm{~K} \\
\left(\text { mdyn } \AA^{2} / \mathrm{rad}\right)\end{array}$ & $\begin{array}{c}\text { Percentage softened } \\
(\%)\end{array}$ \\
\hline Fe-N-C-C & 0.127 & 0.096 & 24.4 \\
N-Fe-N-C & 0.135 & 0.111 & 17.9 \\
Fe-N-C $(-\mathrm{C})$ & 0.062 & 0.050 & 19.4 \\
N-Fe-N(-N) & 0.098 & 0.072 & 16.3 \\
\hline \hline
\end{tabular}

bonds, which are the major dynamical elements contributing to the iron dynamical transition in our model, are softened by only $\sim 20 \%$, as shown in Table I. The difference between the two definitions can be explained with Eq. (1). A simple plot of Eq. (1) (Fig. 5) shows that $r^{2}$ increases exponentially below $50 \mathrm{~cm}^{-1}$. Our results show significant lowering of frequencies in this frequency region. Moreover, we found that atoms with internal coordinates associated with soft bonds exhibit a larger MSD increase than other atoms in one particular normal mode.

The MSD spread over frequency increases disproportionally upon heating, as shown in Fig. 6. At temperatures below $\sim 150 \mathrm{~K}$, the iron MSD for the normal mode frequencies that are below $50 \mathrm{~cm}^{-1}$ takes about $84 \%$ of the total iron MSD, while at $300 \mathrm{~K}$ it increases to $92 \%$.

Generally speaking, the normal modes that participate in biochemical reactions should have the largest motional amplitudes. The largest amplitude among the iron out-of-plane normal modes-normally characterized as the "doming mode" - has been intensively studied experimentally [34,46-48] and theoretically [49-52]. This mode is Raman inactive in a fourfold symmetric porphyrin. The IR spectroscopy and NRVS of cytochrome $f$ failed to identify a wellresolved mode with such a character, and with the intensity expected for a heme doming mode in the low-frequency region. The modes around 40 and $80 \mathrm{~cm}^{-1}$ have been assigned to have the doming features by various authors $[48,51,53]$. In our SCNMA calculation, the normal modes $80 \mathrm{~cm}^{-1}$ have the features of both iron doming motions and in-plane mo-

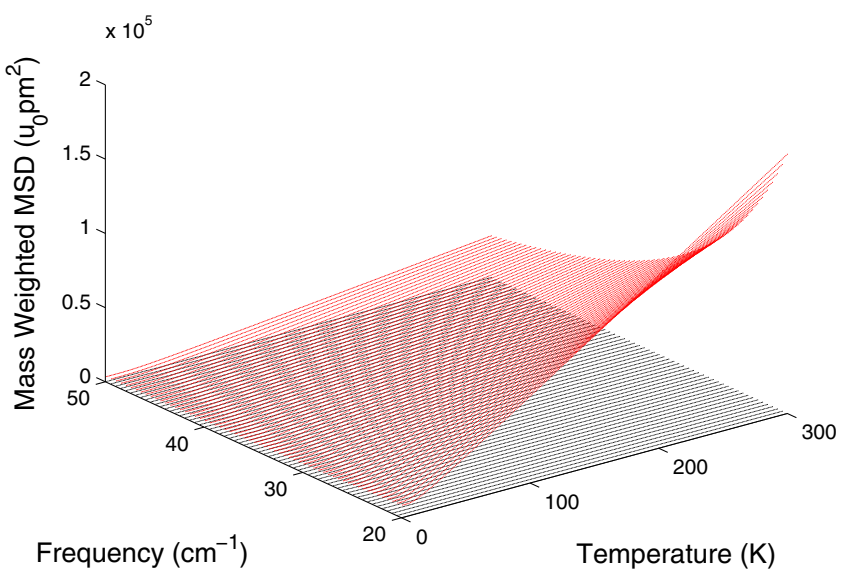

FIG. 5. (Color online) A plot of $\left\langle\sum_{i=1}^{n} m_{i} r_{i}^{2}\right\rangle$ as a function of temperature $(T)$ and (low) frequency $\omega$ from Eq. (1). $u_{0}$ is one atomic weight. 


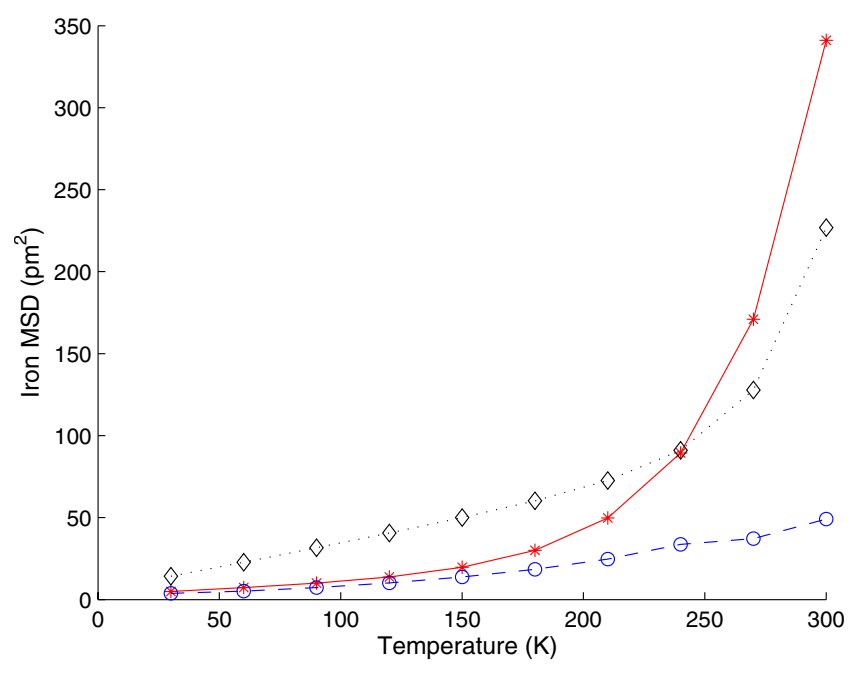

FIG. 6. (Color online) Cyt $\mathrm{f}$ iron MSD from SCNMA in three frequency regimes. (a) Star: $<20 \mathrm{~cm}^{-1}(2.5 \mathrm{meV})$, (b) diamond: $>20 \mathrm{~cm}^{-1}(2.5 \mathrm{meV})$ and $<50 \mathrm{~cm}^{-1}(6 \mathrm{meV})$, and (c) circle: $>50 \mathrm{~cm}^{-1}(6 \mathrm{meV})$.

tions. The iron MSD in the frequency regime 70-90 $\mathrm{cm}^{-1}$ takes less than $10 \%$ of the total MSD, and these modes are close to Gaussian distributions at room temperature from the QHO theory. A theoretical study of the doming mode has been carried out earlier by $\mathrm{Li}$ and Zgierski [49] on a fivecoordinated heme model. In the study, the doming mode was predicted to be around $50 \mathrm{~cm}^{-1}$ and was calculated to be $35 \mathrm{~cm}^{-1}$. Their analysis found that the doming mode takes about $90 \%$ of the iron MSD at room temperature. In one previous NMA calculation, one $37 \mathrm{~cm}^{-1}$ doming mode was found in four-coordinate heme compound $\mathrm{Fe}(\mathrm{OEP})$, which takes $67 \%$ of the total iron MSD (unpublished results). In our six-coordinate cyt $\mathrm{f}$ SCNMA, three normal modes that have the most iron MSD are 19, 35, and $49 \mathrm{~cm}^{-1}$ at low temperature and softened to 14,23 , and $37 \mathrm{~cm}^{-1}$. These three modes take $63 \%$ of the iron MSD and increase to $81 \%$ at room temperature. We assign them to the doming modes due to their significant doming features. The QHO theory indicates that these modes are Gaussian distributions at low temperature $(<100 \mathrm{~K})$ and more classical at room temperature $(300$ $\mathrm{K})$. As temperature increases, these modes develop other features such as saddling and ruffling due to the softening of the dihedral bonds that are associated with these modes. The energy distribution shows that these doming modes are highly delocalized, i.e., the potential energy is distributed among a large number of internal coordinates, and the kinetic energy is distributed among a large number of atoms. We also observe that the iron low-frequency motions are in phase with some other soft bond atoms.

Besides the doming mode, some other significant waterprotein motions are observed in the frequency regime below $50 \mathrm{~cm}^{-1}$. These modes are softened by $20-50 \%$ from low temperature to room temperature. These results can also qualitatively explain the two onsets of anharmonicity suggested by several authors $[9,54]$ as they proposed that there are two motional components: one happens at $T$ of $100 \mathrm{~K}$ and one at $T$ of $200-230 \mathrm{~K}$. As shown in Fig. 6, lowerfrequency modes have a relatively lower dynamical transition temperature.

The statistical properties of fast hydrated protein motions have been analyzed by neutron-scattering [21] and x-ray diffraction [10] experiments. At temperatures below $200 \mathrm{~K}$, the displacement distribution is statistically a Gaussian. However, a deviation from a Gaussian distribution becomes significant at temperatures above $240 \mathrm{~K}$. In our SCNMA calculation, below $100 \mathrm{~K}$, the motions of individual atoms exhibit Gaussian behavior, but starting from $100 \mathrm{~K}$, the atoms participating in soft internal coordinates transition from Gaussian to classical distribution upon heating. The percentage of heavy atoms that exhibit classical behavior rises to $20 \%$ at $300 \mathrm{~K}$. This result agrees with the proposal by other authors who suggested that the protein dynamical transition is caused by water-induced torsional jump $[21,3]$. Furthermore, we also quantitatively identify that the normal modes that contribute to the dynamical transition lie in the frequency regime of $<50 \mathrm{~cm}^{-1}$ at temperatures below the dynamical transition temperature.

\section{CONCLUSION}

SCNMA can be used to study temperature-dependent protein vibrational motions. In the past, all such calculations assumed Gaussian displacement distributions. However, single oscillators depart from Gaussian distribution at higher temperatures. This departure from Gaussian behavior was studied quantitatively here using the QHO theory and SCNMA. Our study of heme c type cytochrome $f$ has led us to identifying some specific features of the atomic interactions which may be of general validity. Our results show that only a few normal modes account for most of the motional amplitudes of a significant set of bonds. These modes lie in the frequency regime $<50 \mathrm{~cm}^{-1}$ (or $<6 \mathrm{meV}$ or $>0.6 \mathrm{ps}$ ). The higher-frequency normal modes essentially maintain a narrow Gaussian distribution. Above $100 \mathrm{~K}$, the low-frequency modes transition from Gaussian to more classical distributions upon heating, facilitating the softening of dihedral (torsional) bonds, which seems to lead to the dynamical transition.
[1] Wolfgang Doster, Stephen Cusack, and Winfried Petry, Nature (London) 337, 754 (1989).

[2] W. Doster, S. Cusack, and W. Petry, Phys. Rev. Lett. 65, 1080 (1990).

[3] M. Ferrand, A. J. Dianoux, W. Petry, and G. Zaccai, Proc.
Natl. Acad. Sci. U.S.A. 90, 9668 (1993).

[4] S. H. Chong, Y. Joti, A. Kidera, N. Go, A. Ostermann, A. Gassmann, and F. Parak, Eur. Biophys. J. 30, 319 (2001).

[5] E. N. Frolov, R. Gvosdev, V. I. Goldanskii, and F. G. Parak, JBIC, J. Biol. Inorg. Chem. 2, 710 (1997). 
[6] K. Achterhold, C. Keppler, A. van Ostermann, U. Bürck, W. Sturhahn, E. E. Alp, and F. G. Parak, Phys. Rev. E 65, 051916 (2002).

[7] W. D. Tian, J. T. Sage, V. Srajer, and P. M. Champion, Phys. Rev. Lett. 68, 408 (1992).

[8] J. van Thor, G. Y. Georgiev, M. Towrie, and J. T. Sage, J. Biol. Chem. 280, 33652 (2005).

[9] Wolfgang Doster, Eur. Biophys. J. 37, 591 (2008).

[10] Dagmar Ringe and Gregory A. Petsko, Biophys. Chem. 105, 667 (2003).

[11] S. Khodadadi, S. Pawlus, J. H. Roh, V. Garcia Sakai, E. Mamontov, and A. P. Sokolov, J. Chem. Phys. 128, 195106 (2008).

[12] Jennifer A. Hayward and Jeremy C. Smith, Biophys. J. 82, 1216 (2002).

[13] Jennifer A. Hayward, John L. Finney, Roy M. Daniel, and Jeremy C. Smith, Biophys. J. 85, 679 (2003).

[14] A. L. Tournier and J. C. Smith, Phys. Rev. Lett. 91, 208106 (2003).

[15] Alexander L. Tourniera, Jiancong Xu, and Jeremy C. Smith, Biophys. J. 85, 1871 (2003).

[16] S. Cusack and W. Doster, Biophys. J. 58, 243 (1990).

[17] Nobuhiro Gō, Tosiyuki Noguti, and Testuo Nishikawa, Proc. Natl. Acad. Sci. U.S.A. 80, 3696 (1983).

[18] Bernard R. Brooks and Martin Karplus, Proc. Natl. Acad. Sci. U.S.A. 80, 6571 (1983).

[19] B. Melchers, E. W. Knapp, F. Parak, L. Cordone, A. Cupane, and M. Leone, Biophys. J. 70, 1043 (2001).

[20] K. Hinsen and G. R. Kneller, Proteins 70, 1235 (2008).

[21] W. Doster and M. Settles, Biochim. Biophys. Acta 1749, 173 (2005).

[22] H.Nakagawa, H. Kamikubo, I. Tsukushi, T. Kanaya, and M. Kataoka, J. Phys. Soc. Jpn. 73, 491 (2004).

[23] H. Nakagawa, H. Kamikubo, and M. Kataoka, Biochim. Biophys. Acta 1804, 27 (2009).

[24] H. Nakagawa, A. Tokuhisab, H. Kamikubo, Y. Jotic, A. Kitaoc, and M. Kataoka, Mater. Sci. Eng., A 442, 356 (2006).

[25] A. Tokuhisa, Y. Joti, H. Nakagawa, A. Kitao, and M. Kataoka, Phys. Rev. E 75, 041912 (2007).

[26] C. J. Carrell, B. G. Schlarb, D. S. Bendall, C. J. Howe, W. A. Cramer, and J. L. Smith, Biochemistry 38, 9590 (1999).

[27] W. Robert Scheidt, Stephen M. Durbin, and J. Timothy Sage, J. Inorg. Biochem. 99, 60 (2005).

[28] E. Prohofsky, Statistical Mechanics and Stability of Macromolecules (Cambridge University Press, New York, 1995).

[29] Y. Gao, K. V. Devi-Prasad, and E. W. Prohofsky, J. Chem. Phys. 80, 6291 (1984).

[30] W. Zhuang, Y. Feng, and E. W. Prohofsky, Phys. Rev. A 41, 7033 (1990).
[31] Y. Z. Chen and E. W. Prohofsky, Phys. Rev. E 49, 3444 (1994).

[32] Earl Prohofsky, Biophysics (Engl. Transl.) 72, 1372 (1997).

[33] B. Frick and D. Richter, Science 267, 1939 (1995).

[34] Dennis D. Klug, Marek Z. Zgierski, John S. Tse, Zhenxian Liu, James R. Kincaid, Kazimierz Czarnecki, and Russell J. Hemley, Proc. Natl. Acad. Sci. U.S.A. 99, 12526 (2002).

[35] Bernard R. Brooks, Robert E. Bruccoleri, Barry D. Olafson, David J. States, S. Swaminathan, and Martin Karplus, J. Comput. Chem. 4, 187 (1983).

[36] Alex D. MacKerell, Jr. et al., J. Phys. Chem. B 102, 3586 (1998).

[37] H. Paulsen, H. Winkler, A. X. Trautwein, H. Grunsteudel, V. Rusanov, and H. Toftlund, Phys. Rev. B 59, 975 (1999).

[38] Wolfgang Sturhahn and A. Chumakov, Hyperfine Interact. 123-124, 367 (1999).

[39] Brajesh K. Rai, Earl W. Prohofsky, and Stephen M. Durbin, J. Phys. Chem. B 109, 18983 (2005).

[40] Brajesh K. Rai et al., J. Am. Chem. Soc. 125, 6927 (2003).

[41] Timo E. Budarz et al., J. Phys. Chem. B 107, 11170 (2003).

[42] Stephen L. Mayo, Barry D. Olafson, and William A. Goddard, J. Phys. Chem. 94, 8897 (1990).

[43] K. L. Adams et al., J. Phys. Chem. B 110, 530 (2006).

[44] Giuseppe Zaccai, Science 288, 1604 (2000).

[45] Bogdan M. Leu et al., Biophys. J. 95, 5874 (2008).

[46] J. T. Sage, S. M. Durbin, W. Sturhahn, D. C. Wharton, P. M. Champion, P. Hession, J. Sutter, and E. E. Alp, Phys. Rev. Lett. 86, 4966 (2001).

[47] Flaviu Gruia, Minoru Kubo, Xiong Ye, Dan Ionascu, Changyuan Lu, Robert K. Poole, Syun-Ru Yeh, and Paul M. Champion, J. Am. Chem. Soc. 130, 13810 (2008).

[48] Flaviu Gruia, Minoru Kuboa, Xiong Yea, and Paul M. Champion, Biophys. J. 94, 2252 (2008).

[49] Xiao-Yuan Li and Marek Z. Zgierski, Chem. Phys. Lett. 188, 16 (1992).

[50] Minoru Kubo, Flaviu Gruia, Abdelkrim Benabbas, Alexander Barabanschikov, William R. Montfort, Estelle M. Maes, and Paul M. Champion, J. Am. Chem. Soc. 130, 9800 (2008).

[51] Pawel M. Kozlowski, Thomas G. Spiro, Attila Beŕces, and Marek Z. Zgierski, J. Phys. Chem. B 102, 2603 (1998).

[52] Pawel M. Kozlowski, Thomas G. Spiro, and Marek Z. Zgierski, J. Phys. Chem. B 104, 10659 (2000).

[53] Bogdan M. Leu, Marek Z. Zgierski, Graeme R. A. Wyllie, W. Robert Scheidt, Wolfgang Sturhahn, E. Ercan Alp, Stephen M. Durbin, and J. Timothy Sage, J. Am. Chem. Soc. 126, 4211 (2004).

[54] E. Cornicchi, M. Marconi, G. Onori, and A. Paciaroni, Biophys. J. 91, 289 (2006). 